\title{
Core Story Creation: Analysing narratives to construct stories for learning Julia Petty
}

\begin{abstract}
Background; Educational research uses narrative inquiry as an approach to gain and interpret people's experiences. An integral component of this research approach is narrative analysis to organise and make sense of acquired narrative in an organised and meaningful way. Core story creation is a method to manage raw data obtained from narrative interviews to construct stories for learning.

Aim: To articulate how core story creation can be used to construct stories from raw narratives obtained by interviewing parents about their neonatal experience, in order to use them to educate learners.
\end{abstract}

Discussion: Core story creation involves reconfiguration of raw narratives illustrated by interviews with a sample of parents of preterm neonates. The process includes listening to and re-reading transcribed narratives, identifying elements of emplotment and re-ordering these to form a constructed story. Thematic analysis is then performed on the story to draw out learning themes informed by the parent participants.

Conclusion: Core story creation using emplotment principles is a strategy of narrative reconfiguration that produces storied constructs. These can be used for resource development and person-centred education about the patient or parent's experience. Implications for practice: Stories constructed from raw narratives in the context of underpinning constructivism can provide a medium or an 'end-product' to use for learning resource development. This can then contribute to the education of students and/or health professionals in relation to learning about, and understanding their patients' experiences through narrative.

Key words: Core story creation Emplotment Narrative analysis 


\section{Introduction}

This paper examines the use of a process known as core story creation as a means of narrative analysis, in relation to an interpretive study that seeks to explore how the narrative of parent's experience of neonatal care can inform person-centred education. The purpose of the paper is to articulate how this process can be used to create stories from the raw narratives obtained from interviews of parents in order to use them in a constructive and meaningful way to educate student nurses and others working in the neonatal field. Firstly, an overview of the constructivist theoretical approach underpinning this interpretive, narrative based inquiry is given followed by a brief synopsis of the study and conceptual framework. An analysis will then follow of the process of core story creation in relation to where it is placed within narrative analysis as a whole and the implications to practice for researchers undertaking this type of research who can apply these principles to nurse education.

\section{Underpinning theoretical framework}

The methodological approach taken in the present study is narrative inquiry; this is research that focuses on personal experience and takes account of the relationship between individual experience and context (Allen, 2006; Kaplan-Myrth, 2007). As a result, qualitative research of this nature is considered appropriate when research seeks to discover an in-depth understanding of individuals' lived experiences and is valued as an approach in health research when we seek to explore and understand the patient experience (Petty, 2016 a \& b). One way of course to do so is to listen to patient stories which have been shown to elicit thought, reflection and enhance the learning experience in relation to humanistic understanding (Cole, 2009). Frank (2013) , in an 
account of his own illness aimed to amplify and connect voices that told stories about suffering, offering the health professional a view from the patient's perspective.

Narrative research sits within the interpretive paradigm where the notion of interpretation means the discernment of meaning. Charon (2007), a prolific writer on the concept of narrative medicine proposes that- human beings .... 'absorb, interpret, and respond to stories' ... resulting in narrative knowledge. This is fitting with the perspective of constructivism where meaning making is central and an emphasis is placed on creation and sharing of knowledge. Bruner's 'narrative construction of reality' speaks of narrative 'structuring' and how story making is central to creating an understanding of the world and people within it (Bruner, 1991).

There is no chronological order to three concepts: that of narrative inquiry, interpretivism, and constructivism; all work together as one closely connected framework (Figure 1). Narrative is the starting point, by which we then interpret experience and by doing do construct meanings and knowledge, remembering at all times in the context of the present study, the parents at the centre. It is after all, their unique, personal experiences and narratives that form the basis of all subsequent analysis in the study, as discussed in this paper. It is important not to lose sight of the importance person-centred approach to care when researching people's experience. 


\section{Figure 1: Theoretical perspective}

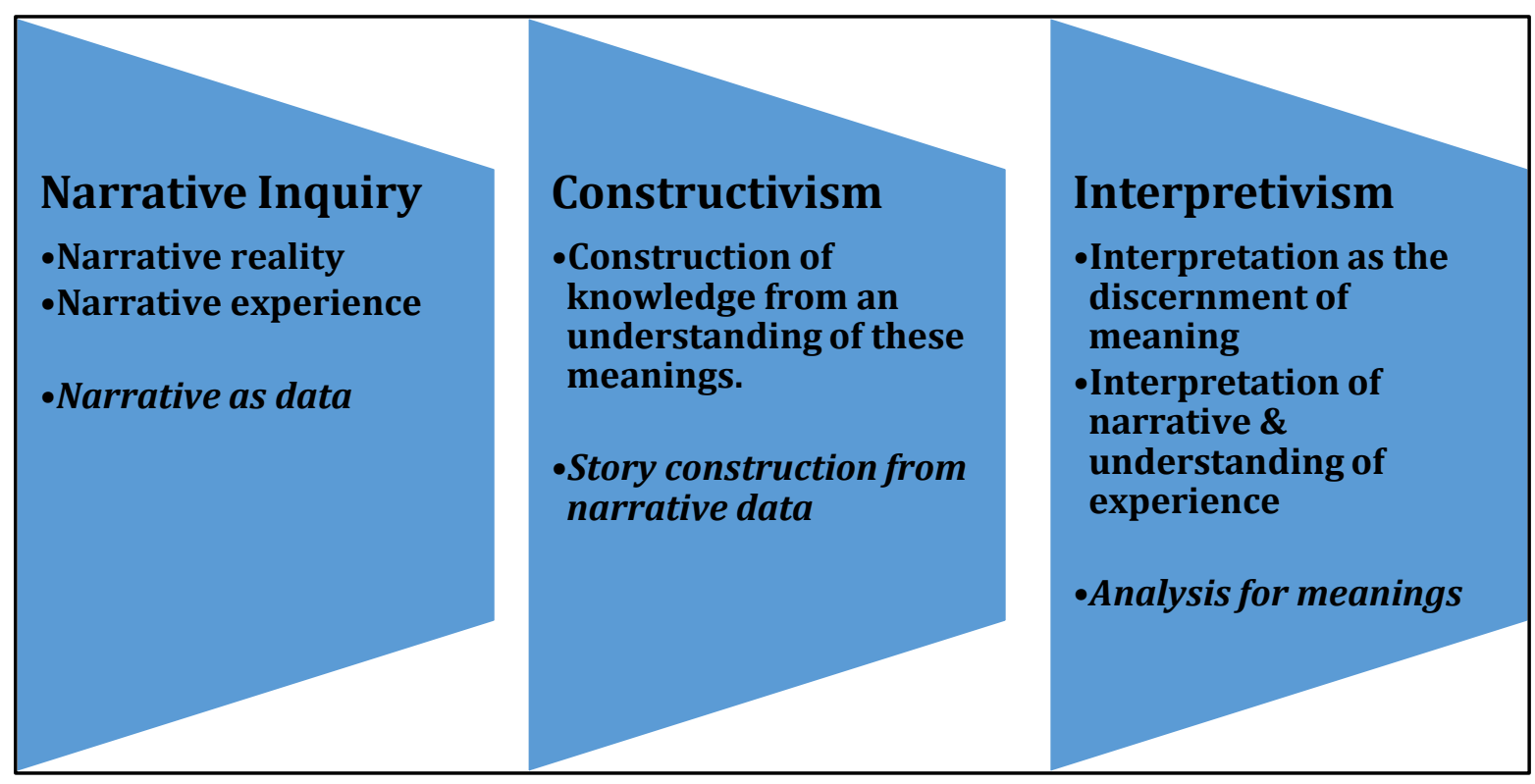

To summarise in the case of the present study, we start with parent's narrative. From that, we construct stories and then interpret these for meanings to be used to impart knowledge and a person-centred understanding of those in our care.

\section{Study overview and conceptual framework}

The study is an interpretive, narrative based study that firstly, aims to create stories from the interviews with parents of preterm neonates. It then aims to explore how these stories of early birth and the subsequent neonatal care experience can be used to effectively educate children's nursing students about the humanistic side of caring for neonates and families. The purpose of interpretive work in the context of this study then is to assign meaning to parent narratives to acquire knowledge about their experience to learn from. The three phases of the study are organised within a conceptual framework derived from Charon's (2007) 'Narrative medicine' work that views narrative in terms of attention, representation and affiliation. To explain, phase 1 
is Attention to narrative where parent stories are obtained and constructed from narrative interviews. Phase 2 is Representation of narrative where said stories are developed into a digital platform so representing the created stories. Finally, phase 3 is Affiliation to narrative where the 'connectedness' of narrative to person-centred, empathic understanding and learning will be explored. Phases 2 and 3 are reliant on the initial phase 1, emphasising the importance of both the raw experiences of parents and the story creation stage in order to produce a manageable narrative 'product' to work with for the remainder of the study. The study will continue to use the stories as a basis for exploration of their value for person-centred teaching and learning. The discussion that now follows in this paper however, focuses on phase 1.

\section{'Attention' to narrative': Core Story creation}

Phase 1 involved the collection of narrative from parents and the subsequent data analysis in order to generate themes and to create stories that will be used for later resource development. The methodological process has been informed by the undertaking of a small scale study using interviews and story creation with student nurse participants (Petty, 2016a).

\section{Narrative interviews}

Initially, twenty parent participants were recruited, selected by purposive sampling on a volunteer basis through BLISS baby charity and other external contacts. Narrative interviews were undertaken, an unstructured form of interviewing which encourages and stimulates interviewees to tell a story about a significant event in their life. They started with an open 'single question aimed inducing narrative' (SQUIN) as offered by Wengraf (2009). A SQUIN is deliberately broad aiming to provide a means of 
stimulating dialogue allowing participants to construct their narrative on their own, in this case to enable the parents to talk through their whole experience or 'journey' on the neonatal unit. The SQUIN asked was " Can you tell me about your experience while you and your baby were on the neonatal unit, taking me through the events and experiences that were important for you, from admission to discharge? Narrative interviewing is also congruent with the overarching narrative paradigm where the focus was to gain stories, rather than a prescriptive, structured form which only sought closed responses.

All interviews were audio recorded using a voice recorder and the audio files were later transcribed manually. Verbatim transcription was undertaken by the author themselves, due to the issues of confidentiality. The need for transcription has been discussed in the literature. Halcomb and Davidson (2006) raises the debate on the necessity for transcription of all verbatim audio recorded interview data and Reissman (2008) suggests that transcription is actually not always necessary. However, Stuckey (2014) see that moving from raw interviews to valid interpretations requires preparing transcripts to enable this. Oliver et al (2005) see transcription is a powerful act of representation. For the present study, the decision was made to manually transcribe from audio to verbatim written formats to make an 'end-product' that was tangible with which to work with. In other words, verbatim transcriptions provided the raw data in text form, necessary for later story creation. While it was time consuming, this also enabled an immersion and familiarisation with the content on the part of the researcher. 


\section{Story creation \& emplotment}

Following transcription, the next stage was to work with the raw narrative to create the stories. Limited research in health has offered strategies for how stories can be constructed from narratives collected from patient interviews about their experiences. Emden (1998) describes the process of 'core story creation', a process to condense full length narratives or stories to briefer ones, to facilitate the narrative analysis process. Her work is based on both Polkinghorne's $(1988,1995)$ and Mishler's $(1995)$ models who have used similar methods to analyse narratives and to re-construct events into a form of plot. This is defined as 'emplotment' whereby a series of events is assembled into a narrative with a plot. In the health literature, this has also been referred to as therapeutic 'emplotment' which Mattingly (1994) defines as the making of story-like structures through interactions between a therapist and their client which encourages the former to view the healing process. Crossley (2003) concurs and states 'therapeutic emplotment' is implicitly incorporated by the patient, providing an underlying plot structure to his story" (pg. 439). Tropea (2012) sees therapeutic emplotment as a way of improving connections with patients and that narratives can be constructed together. Such a definition places emplotment within the constructivist framework. As Mishler (1995) states, analysis in this way is 're-constructing the told from the telling'.

In this study, core story creation involved creating stories from the transcribed raw narratives within a constructivist theoretical approach. This method of analysis is also influenced by Bruner's (1991) narrative 'structuring' in which data collected consists of actions, events and happenings. The subsequent data analysis involves their synthesis and configuration to produce or construct stories as one of the outcomes of the research 
process. The notion of emplotment therefore is akin to what Bruner (1991) terms 'story making' as referred to in an earlier section.

Emplotment or in other words, plot creation is also a way to manage and organise narrative, a principle relevant to the present study. Limited research has used plot creation to organise narratives gained from interviews. For example, studies by Haidet et al, (2006) and Kelly and Howie (2006) used emplotment principles to enable the researchers to work with, re-configure and give structure to raw narrative in the form of storied constructs. The present study used such a process and re-ordered the raw data into an altered form, outlined in Figure 2: Core Story Creation.

Overall, it has been illustrated here, how a specific process can be used to construct stories from the re-configuration of raw narratives of parents. One may ask whether this process could change the meaning held and why is it necessary to change the raw data as that is what the parent or participant has said. However, rationale is proposed by Kim, (2015) and Mishler (1995) and their similar views of reconstructing the 'told from the telling' or, reordering a storyline to be chronologically organised. Participants may not tell their stories in an order that makes sense to the researcher and they may not be ordered thematically or temporally. They may deviate or focus on a topic that is irrelevant or they may go backwards and forwards in time intermittently. Therefore, to reconstruct the order from the 'telling' makes the story more coherent leading to the reconstructed storyline becoming the 'narrative for further analysis' (Mishler, 1995). 
Figure 2: Core story creation

(Adapted from Emden, 1998; Kelly and Howie, 2007; Petty, 2016a)

\section{RAW NARRATIVE OBTAINED FROM PARTICIPANT INTERVIEWS}

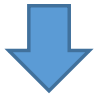

1-Interview transcripts read several times to absorb and understand the content

2-All interviewer contributions deleted from the transcript.

*3-All unnecessary words or sentences that detract from the key idea of the participants' sentences are deleted.

*4-Remaining text re-read for sense.

5-Text re-configured - i.e. divided into events which are then re-ordered chronologically. The end product is now a series of events ('emplotment')

6-Cross checking between the re-configured text and the original transcripts in order to ensure meanings are re-validated

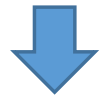

7-The events / plots are combined to create a single coherent story for each participant in chronological order.

8-Verification by returning the transcripts and stories to the participants. If necessary, make final changes / corrections

\section{CORE STORY CREATION COMPLETE}

*Steps 3 and 4 repeated so only key ideas are retained.

\section{Verification}

Before continuing with subsequent analysis, verification of the stories for credibility with participants themselves is the final stage of Emden's approach to core story creation. The transcripts along with the created stories were sent back to the parents in 
this study so that the authentic representations of what they had said can be substantiated against their own observations of the same episode, offering opportunities to alter, add or remove anything from their stories. This process is based on principles outlined by Spalding \& Phillips (2007) to address trustworthiness of the data and interpretation, in line with optimising credibility of a study.

\section{Thematic analysis}

Narrative analysis is one type of qualitative analysis that explores the data specifically looking for stories (Lichtman, 2012). In this study, final analysis of the narrative, already in storied form, was necessary in order to extract meanings and to generate themes from the data. This was undertaken using Braun and Clarke's (2006) thematic analysis framework. Thematic analysis focuses on common, emergent themes extricated from raw data closely linked with that used in other qualitative research approaches such as phenomenology (Reissman, 2008). It is most suited for the current study rather than other forms of analysis (structural, discourse, visual) as this study is concerned with the 'what' of a story rather than 'how' it is told; in other words, the content of a story. Themes are patterns across data sets that are used to identify important areas for further discussion and learning. The rationale for performing thematic analysis after the story creation stems from the fact that the created story becomes the unit of further analysis (Kim, 2015; Mishler, 1995). This meant the parents' stories were used to understand the meanings that are embedded in experiences that people, or in this case parents, relate.

Rationale for the use of Braun and Clarke's model for thematic analysis arose from it's lucidity and clear, staged approach which also enabled main themes and sub-themes to 
emerge. While other frameworks do exist, this one served as a workable and feasible approach to identification of a range of common themes as was the case in the pilot study (Petty, 2016a). See Figure 3 which outlines the thematic analysis process as it applies to the analysis of stories created from the preceding core story creation, undertaken in the present study.

Figure 3: Thematic Analysis applied to Core Story Creation STORIES<smiles>C1C2CC1C1CC2C1</smiles>

Familiarising with the stories by transcribing, reading, rereading it and noting down initial ideas.

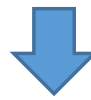

Generating initial codes: Coding interesting features across the entire story collection and organising data relevant to each code.



Searching for themes: Sorting codes into potential themes and gathering all data relevant to each potential theme.

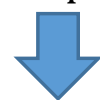

Reviewing themes: assigning main and sub-themes to enable categorisation.

Defining and naming themes: Refining the specifics of each theme, and the overall story the analysis tells; generating clear definitions and names for each theme.

(Adapted from Braun \& Clarke, 2014)

*THEMES

- The physical and emotional needs of parents

- The psychological impact of the neonatal experience

- The effect of separation between parents and baby

- The need for communication and information 
There are some key emergent themes that have arisen from the thematic analysis of the parent stories within the current study. It is not in the remit of this paper to outline these in detail and will be the subject of a future, separate paper. However, it is worth highlighting how core story creation sets up subsequent analysis and the generation of key themes for learning. These key themes and messages from parents are vitally important areas in relation to their potential role in informing person-centred education, relevant to moving forward to phases 1 and 2 of the study.

\section{Implications for practice}

In essence, what this paper has discussed is the creation of stories for learning. By this, it can be seen how narrative has the power to educate and transform thought, in that it is used to explore and understand experience. Narrative inquiry then, in a teaching and learning context focuses on how individuals make sense of their world, experience and self from which themselves or others can learn (Price, 2011; Riley \& Hawe, 2005). This is relevant to the present study in that it is interested in how learners and/or health professionals make sense of what parents experience to learn about neonatal care, again remembering the parents at the centre. Interpretivism is integral to this process: this involves interpreting narrative and the experiences spoken about to arrive at meanings integrating human interest (Creswell, 2014; Holloway \& Biley, 2011; Holloway \& Freshwater, 2007; Kaplan-Myrth, 2007).

In the context of health, stories of illness provide a medium for the education of both patients and health professionals and this may provide a way of connecting the different worlds of both. From an epistemological viewpoint, enhancing narrative knowledge from 
creating stories leads to deeper understanding about a situation or person who is vulnerable, ill, under the care of professionals or, in this case, the family or carers of the patient. As a result, the outcome of a narrative approach to producing stories is not the generalisation of pure objective, scientific data but a more profound understanding of experiences from the perspectives of patients and in the case of research methodology, the participants (parents) selected for a study (Johnson, 2009). This is congruent with a humanistic, person-centred healthcare system that places human experience at the heart of care (Charon, 2001; Charon, 2007; DasGupta, 2007; Divinsky, 2007; Hall \& Powell, 2011).

The potential pedagogical value of story creation is vast. Literature supports this and illustrates how stories can enable us to gain insight into the lived experiences of patients and families (Christiansen, 2011; Rashotte, 2005). Engaging with these stories enables a development of understanding of the healthcare experience (Fenton, 2014). In addition, "there is a growing realization that patients and service users are a rich source of healthcare-related stories that can affect, change and benefit clinical practice" (Haigh \& Hardy, 2011: pg. 411).

Themes have already emerged in the current study that one can instantly see are vitally important for learners and health professionals caring for neonates and their families to take note of, to better understand patient / parent experience and therefore in the long run, offer more person-centred care (Petty, 2016b). Moreover, storied constructs provide us with an end-product that can be packaged up to create resources most commonly digital in format, that may be disseminated for learning purposes in relation to enhancing person-centred understanding through narrative. Again, research that has 
evaluated the effect of such digital storytelling has highlighted their value in providing an effective medium that can be used to capture and share knowledge, enhance reflective learning and promote a better understanding of the patient (Price et al, 2015). Digital storytelling therefore integrates the strengths of narrative and technology. To fully appreciate the learning potential of digital storytelling, stories must be shared among learners, and individuals need opportunities to use and reflect on them. The stories constructed in the present study will now be used to do just this; to develop a resource which will be disseminated to learners for them to learn about the neonatal experience from the perspectives of parents.

\section{Conclusion}

This paper has discussed how core story creation using emplotment principles can be used to construct stories from raw narratives obtained by interviewing parents about their neonatal experience, in order to use them to educate learners. The experience of the parents that has informed the analysis processes and indeed the remainder of the study as a whole, must be kept in kind throughout. After all, the core interest is personcentred education. Within the context of constructivism, the processes discussed can provide a basis for learning resource development. This can then contribute to the education of students and/or health professionals in relation person-centred understanding of their patients and their experiences through narrative. 


\section{References}

Allen, C. (2006). Narrative Research. Nurse Researcher, 13, 3, 4-6.

Braun, V., \& Clarke, V. (2014). What can thematic analysis offer health and wellbeing researchers? International Journal of Qualitative Studies on Health and Well-Being, $9,1-2$.

Bruner, J. (1991). The Narrative Construction of Reality. Critical Inquiry, 18, 1, 1-21.

Charon, R. (2001). Narrative Medicine A Model for Empathy, Reflection, Profession, and Trust. JAMA, 286, 15, 1897-1902.

Charon, R. (2007). What to do with stories. Canadian Family Physician, 53, 8, 12651267.

Christiansen, A. (2011). Storytelling and professional learning: A phenomenographic study of students' experience of patient digital stories in nurse education. Nurse Education Today, 31, 3, 289-293.

Cole, M. (2009). Storytelling: its place in infection control education. Journal of Infection Prevention, 10, 5, 154-158.

Creswell, J. (2014). Research Design: Qualitative, quantitative and mixed method approaches (4th edition). Sage: Los Angeles and London.

Crossley, M. L. (2003). 'Let me explain': narrative emplotment and one patient's experience of oral cancer. Social Science \& Medicine, 56, 3, 439-448.

DasGupta, S. (2007). Between stillness and story: lessons of children's illness narratives. Pediatrics, 119, 6, e1384-91.

Divinsky, M. (2007). Stories for life: introduction to narrative medicine. Canadian Family Physician, 53, 2, 203-5.

Emden, C. (1998). Conducting a narrative analysis. Collegian (Royal College of Nursing, Australia), 5, 3, 34-39.

Fenton, G. (2014). Involving a young person in the development of a digital resource in nurse education. Nurse Education in Practice, 14, 1, 49-54.

Frank, AW (2013). The Wounded Storyteller- Body, Illness and Ethics (2nd edition). University of Chicago Press. Chicago and London.

Haidet, P., Kroll, T. L., \& Sharf, B. F. (2006). The complexity of patient participation: Lessons learned from patients' illness narratives. Patient Education and Counseling, 62, 3, 323-329.

Haigh, C., \& Hardy, P. (2011). Tell me a story - a conceptual exploration of storytelling in healthcare education. Nurse Education Today, 31, 4, 408-411.

Halcomb, E. J., \& Davidson, P. M. (2006). Is verbatim transcription of interview data always necessary? Applied Nursing Research, 19, 1, 38-42.

Hall, J. M., \& Powell, J. (2011). Understanding the Person through Narrative. Nursing Research and Practice, 1-10. http://doi.org/10.1155/2011/293837 
Holloway, I., \& Freshwater, D. (2007). Narrative Research in Nursing. Blackwell. Chichester.

Holloway I. \& Biley, F. (2011). Being a qualitative researcher. Qualitative Health Research, 21, 968-975.

Johnson, N. (2009). The role of self and emotion within qualitative sensitive research : a reflective account. Enquire, 2, 4, 23-50.

Kaplan-Myrth, N. (2007). Interpreting people as they interpret themselves: Narrative in medical anthropology and family medicine. Canadian Family Physician. 53, 8, 12681269.

Kelly, T \& Howie, L. (2007). Working with stories in nursing research: Procedures used in narrative analysis: Feature article. International Journal of Mental Health Nursing, 16, 2, 136-144.

Kim, J. (2015). Narrative Data Analysis and Interpretation: Flirting with Data. Chapter 6, in Understanding Narrative Inquiry: The crafting and analysis of stories as research. Sage. Singapore (pp. 185-224).

Lichtman, M. V. (2012). Making Meaning from your data. In Qualitative Research in Education: A User's Guide. Sage, London.

Mattingly, C. (1994). The concept of therapeutic "emplotment". Special issue; narrative representation of illness and healing. Social Science and Medicine, 38, 6, 811-822.

Mishler, E. (1995). Models of Narrative Analysis: A Typology. Journal of Narrative and Life History, 5, 2, 87-123.

Oliver, D. G., Serovich, J. M., \& Mason, T. L. (2005). Constraints and Opportunities with Interview Transcription: Towards Reflection in Qualitative Research. Social Forces; a Scientific Medium of Social Study and Interpretation, 84, 2, 1273-1289.

Petty, J. (2016a). Creating stories for learning about the neonatal care experience through the eyes of student nurses: an interpretive, narrative study. Nurse Education Today. 48, 25-32.

Petty, J. (2016b). Learning from narrative to understand the person-centred experience in neonatal nursing: A literature review. Journal of Neonatal Nursing. 22, 6, 297308.

Polkinghorne, D. (1988). Narrative Knowing and the Human Sciences. University of New York Press, Albany.

Polkinghorne, D. (1995). Narrative configuration in qualitative analysis. In R. Hatch, R \& Wisniewski (Ed.), Life History and Narrative. Falmer Press, London \& Washington.

Price, B. (2011). Improving clinical reasoning in children's nursing through narrative analysis. Nursing Children and Young People, 23, 6, 28-35.

Price, D. M., Strodtman, L., Brough, E., Lonn, S., \& Luo, A. (2015). Digital Storytelling: An Innovative Technological Approach to Nursing Education. Nurse Educator, 40, 2, 66-70.

Rashotte, J. (2005). Dwelling with stories that haunt us: Building a meaningful nursing 
practice. Nursing Inquiry, 12, 1, 34-42.

Reissman. CK. (2008). Thematic analysis. Chapter 3, in, Narrative Methods for the Human Sciences. Sage, London \& California (pp. 53-76).

Riley, T., \& Hawe, P. (2005). Researching practice: The methodological case for narrative inquiry. Health Education Research, 20, 2, 226-236.

Spalding, N. J., \& Phillips, T. (2007). Exploring the Use of Vignettes: From Validity to Trustworthiness. Qualitative Health Research, 17, 7, 954-962.

Stuckey HL. (2014) The first step in Data Analysis: Transcribing and managing qualitative research data. J Soc Health Diabetes. 2, 6-8

Tropea, S. (2012). "Therapeutic emplotment": A new paradigm to explore the interaction between nurses and patients with a long-term illness. Journal of Advanced Nursing, 68, 4, 939-947.

Wengraf, T. (2009). Qualitative Research Interviewing- Biographic Narrative and Semi structured Methods. Sage, London. 